\title{
Ex Vivo expansion of suboptimal (low dose) cord blood products provides sufficient cells for transplantation of adult patients
}

\begin{abstract}
Background: Recent trials have demonstrated the potential to combine two cord blood $(\mathrm{CB})$ products to increase the cell dose delivered, however often $\mathrm{CB}$ products with sufficient cell dose, have a 2 antigen mismatch to each other or the recipient. Better matched products are routinely available however, their use is limited due to low cell content. We have developed ex vivo expansion techniques that can provide a 10 to 20 fold increase in cell numbers and evaluated the potential of ex vivo expansion to provide sufficient doses for these suboptimal products.
\end{abstract}

Methods: CB products which were frozen for banking and rejected due to low cell doses (median cells dose $0.7 \times 10^{7}$ total nucleated cells $(\mathrm{TNC}) / \mathrm{kg}$ for a $50 \mathrm{~kg}$ individual) were expanded using co culture on allogeneic mesenchymal stem cells (MSC). The CB products were thawed and the mononuclear cell (MNC) fraction isolated by density gradient centrifugation. The CB MNCs were cultured in $\mathrm{T} 162 \mathrm{~cm}^{2}$ tissue culture flasks pre established with MSC in expansion media plus SCF, G-CSF and Tpo. After culture for 2 weeks the $\mathrm{TNC} / \mathrm{kg}$ were determined.

Results: A median fold expansion of 9 fold was obtained $(\mathrm{N}=5)$ resulting in a median cell dose post expansion of $4.3 \times 10^{7} \mathrm{TNC} / \mathrm{kg}$ (for a $50 \mathrm{~kg}$ individual). In addition, fractionation of the CD34+ve and CD34-ve subsets from the CB MNC, demonstrated that the expansion was almost exclusively from the CD34+cells.

Discussion: These data demonstrate that co culture of CB MNCs on MSC result in significant expansion of suboptimal CB products. The expanded CB products would meet the minimal cell requirements for use of $\mathrm{CB}$ in adults and therefore provide the potential use of better matched CB products. This could result in enhanced engraftment and less toxicities such as graft versus host disease that occurs with lower level HLA matched $\mathrm{CB}$ products.

Keywords: cord blood, ex vivo expansion, CD34+ cells
Volume I Issue I - 2015

\author{
lan McNiece, Santhosh Silvajothi, Joshua \\ Kellner \\ Department of Stem Cell Transplantation and Cellular Therapy,
}

The University of Texas MD Anderson Cancer Center, USA

Correspondence: lan McNiece, PhD, Department of Stem Cell Transplantation and Cellular Therapy, The University of Texas MD Anderson Cancer Center, 1515 Holcombe Blvd, unit 0065, Houston, TX 77030, USA,

Email imcniece@mdanderosn.org

Received: April 28, 2015 | Published: May 19, 2015

\section{Introduction}

The use of umbilical cord blood (CB), as a source of hematopoietic cells for patients undergoing high dose chemotherapy, has become a standard therapeutic option for pediatric patients with hematologic malignancies. ${ }^{1-5}$ However, the low cell doses in CB products has limited the use in adult patients due to delayed engraftment and survival. ${ }^{6,7}$ This has led to evaluation of approaches to increase the cell dose by ex vivo expansion. ${ }^{8-10}$ Several groups have evaluated the potential of ex vivo expanded $\mathrm{CB}$ units to enhance engraftment, ${ }^{8-10}$ however, the results to date have failed to demonstrate significant improvement in the time to neutrophil engraftment. We have proposed that current methodologies employed in these trials, which include selection of $\mathrm{CD} 34+$ or CD133+ cells from frozen CB units, are problematic due to significant cell loses during selection and low purities in a number of products. ${ }^{10,11}$ Based upon the issues associated with selection of frozen $\mathrm{CB}$ units, we have developed culture methods for co culture of $\mathrm{CB}$ mono nuclear cells (CB-MNC) on mesenchymal stem cells (MSC). ${ }^{11}$ We have demonstrated an expansion of total nucleated cells (TNC) of 10 to 20 fold and clinical trials have demonstrated decreased time to neutrophil and platelet engraftment. ${ }^{12}$

Another approach that has been explored for delivering higher cell doses with CB products is the use of two CB products. ${ }^{13}$ Wagner and colleagues have demonstrated the feasibility of this approach for use in adults; however the majority of recipients receive 4 of 6 HLA CB products. A number of patients have better matched CB products available (5 of 6 or 6 of 6 ), however these products have low cell doses $\left(<2 \times 10^{7} \mathrm{TNC} / \mathrm{kg}\right)$ and are not suitable for use. We hypothesized that the co culture expansion conditions we developed could be suitable to expand suboptimal $\mathrm{CB}$ products, i.e. products with cell doses below $2 \times 10^{7} \mathrm{TNC} / \mathrm{kg}$, to provide a sufficient cell dose for adult patient and the use of better matched products would result in decreased immunological complications including graft versus host disease (GVHD).

\section{Methods}

\section{Cord blood products}

Frozen CB products: Frozen CB products were supplied by Joanne Kurtzberg (Duke University, Durham, NC). The products had been frozen for banking however they were deemed unsuitable due to low cell numbers. All products were collected under a Duke University IRB approved protocol with signed consent. The products were shipped in a dry shipper and stored in liquid nitrogen until used. 
Fresh CB products: Fresh CB products were supplied by Dr. Pablo Rubenstein (New York Cord Blood Bank, New York, NY) and were shipped overnight at room temperature. These products were collected for banking under appropriate IRB approval and signed consent but were deemed unsuitable for banking due to low cell numbers or other issues. CB-MNC was isolated from fresh CB products by density centrifugation over ficoll and the low density cells recovered. $\mathrm{CD} 34+$ cells were isolated from the CB-MNC using the Vario MACS selection device (Miltenyi Biotech, Cologne, Germany). Expansion was performed on MSC as described below in Stemline II media plus growth factors.

\section{Isolation of MSC}

Bone marrow cells were purchased from Allcells Inc (Emeryville, $\mathrm{CA}$ ) who obtained the $\mathrm{BM}$ aspirates from normal donors under appropriate IRB approvals. The MNC fraction was isolated by ficoll separation and MSC were grown to confluency in $\mathrm{T} 162 \mathrm{~cm}^{2}$ tissue culture flasks (Corning, Acton, MA) in alpha MEM plus 20\% FCS.

\section{Ex Vivo expansion of CB-MNC on MSC}

$\mathrm{CB}$ products were thawed rapidly in a water bath and the $\mathrm{MNC}$ fraction isolated by density separation over a gradient and the low density cells collected and resuspended in $500 \mathrm{ml}$ of Stemline II expansion media (Sigma Aldrich, St Louis, MO) containing 100ng/ml each of recombinant human (rh) stem cell factor (rhSCF), granulocyte colony stimulating factor (rhG-CSF) and thrombopoietin (rhTpo). Ten (10) T162 $\mathrm{cm}^{2}$ cultureflasks containing confluent layers of MSC were prepared by removing the growth media (alpha MEM plus $20 \%$ FCS) and replacing with $50 \mathrm{ml}$ of $\mathrm{CB}$ MNCs in media plus growth factors, representing $10 \%$ of the $\mathrm{CB}$ products. The cultures were incubated for 7 days in a $5 \% \mathrm{CO}_{2}$ incubator at $37^{\circ} \mathrm{C}$. After 7 days the non adherent cells were removed from the flasks and transferred to a 1 liter Teflon bag (American Fluoroseal Corp, Gaithersburg, MD) with an additional $350 \mathrm{ml}$ of media and growth factors added. Fresh media and growth factors $(50 \mathrm{ml})$ were placed in the T162 flasks. After an additional 7 days (14 days total incubation) both the bags and flasks were harvested and the cells pooled.

\section{Flow cytometric analysis}

Cells were analyzed for phenotypic expression of surface proteins specific for subpopulations of hematopoietic cells. Approximately 1 million cells were stained with anti-CD34-PE and anti-CD45-FITC (Becton-Dickinson, San Jose, CA) and a minimum of 50,000 events collected in a list mode file format by flow cytometry (FACS Vantage, Becton-Dickinson). Aliquots of cells were also stained with isotype control antibodies.

\section{Results}

\section{Expansion of CB mononuclear cells on MSC}

$\mathrm{CB}$ products were thawed and washed, resulting in a median of $3.3 \times 10^{8} \mathrm{TNC}$ (range 1.4 to $3.6 \times 10^{8}$ ). For a $50 \mathrm{~kg}$ recipient, these $\mathrm{CB}$ products would provide only $0.73 \times 10^{7} \mathrm{TNC} / \mathrm{kg}$ with zero of 5 products reaching the minimal target dose of $1 \times 10^{7} \mathrm{TNC}$. These products may be well matched for a patient based upon HLA, but would not be suitable for clinical use due to the low cell dose and so decreasing the number of available grafts for patients. In many cases more mismatched products must be used and so increasing the potential toxicities due to GVHD or the potential of rejection.
Each product was expanded by culturing the MNC fraction on preformed layers of MSC. Ten (10) T162 $\mathrm{cm}^{2}$ flasks were used for each product such that each flask contained $10 \%$ of the CB MNC. After ex vivo culture a median of 9 fold expansion of TNC was obtained with a range of 6.5 to 24 fold. The median TNC post expansion was $21.6 \times 10^{8}$ (range 11 to $79 \times 10^{8} \mathrm{TNC}$ ) (Table 1 ). For a $50 \mathrm{~kg}$ recipient, the expanded $\mathrm{CB}$ product would be equivalent to $4.3 \times 10^{7} \mathrm{TNC} / \mathrm{kg}$ (range 2.2 to $16 \times 10^{7}$ ), with all 5 expanded products reaching the minimal target of $1 \times 10^{7} \mathrm{TNC} / \mathrm{kg}$ (Table 2). In fact all expanded products contained more the $1 \times 10^{7} \mathrm{TNC} / \mathrm{kg}$ based upon a $100 \mathrm{~kg}$ recipient.

Table I Ex Vivo Expansion of TNC from frozen CB products

\begin{tabular}{lcccccc}
\hline TNCx 10 & & & & & & \\
\hline Exp \# & $\mathbf{I}$ & $\mathbf{2}$ & $\mathbf{3}$ & $\mathbf{4}$ & $\mathbf{5 M e d i a n}$ & \\
\hline CB-MNC & 3.6 & 2.2 & 1.4 & 3.3 & 3.3 & 3.3 \\
Post expansion & 44.6 & 19 & 11 & 21.6 & 79.3 & 21.6 \\
Fold expansion & 12 & 9 & 7 & 6.5 & 24 & 9 \\
\hline
\end{tabular}

Table 2 Cell doses based upon recipient weight of $50 \mathrm{~kg}$ (A) and 100kg (B)

$$
\text { TNC } \times 10^{7} / \mathrm{kg}
$$

A: Recipient Weight $50 \mathrm{~kg}$

\begin{tabular}{lllllll}
\hline Exp \# & $\mathbf{I}$ & $\mathbf{2}$ & $\mathbf{3}$ & $\mathbf{4}$ & $\mathbf{5 M e d i a n}$ & \\
\hline CB-MNC & 0.7 & 0.4 & 0.3 & 0.7 & 0.7 & 0.7 \\
Post Expansion & 8.9 & 3.8 & 2.2 & 4.3 & 15.9 & 3.8 \\
\hline
\end{tabular}

B: Recipient Weight $100 \mathrm{~kg}$

\begin{tabular}{lllllll}
\hline Exp \# & $\mathbf{1}$ & $\mathbf{2}$ & $\mathbf{3}$ & $\mathbf{4}$ & 5Median & \\
\hline CB-MNC & 0.4 & 0.2 & 0.1 & 0.3 & 0.3 & 0.3 \\
Post Expansion & 4.5 & 1.9 & 1.1 & 2.2 & 7.9 & 2.2
\end{tabular}

\section{Expansion of CB CD34+ Cells on MSC}

We hypothesized that the major cell population in the $\mathrm{CB}$ units responsible for expansion would be CD34+ cells. To test this hypothesis we fractionated $\mathrm{CB}$ MNC into CD34+ and CD34fractions. $\mathrm{CB} \mathrm{MNC}, \mathrm{CB} \mathrm{CD} 34+$ and $\mathrm{CB}$ CD34- cell populations were then cultured on preformed layers of MSC. The culture of CB MNC on MSC resulted in a median of 15 fold expansion of TNC with a total of $3.4 \times 10^{8}$ cells harvested after 14 days of culture (Table 3 ). There was a median of 29 fold expansion of CD34+ cells. Co-culture of CD34+ cells on MSC resulted in a median of 1,352 fold expansion of TNC with a median expansion of 54 fold of CD34+ cells. The CD34-ve fraction resulted in low levels of expansion with a 3 fold expansion of TNC and a 4 fold expansion of CD34+ cells. The levels of TNC and CD34+ cells harvested from the CB MNC cultures was approximately equal to the combination of cells harvested from the CD34+ fraction and the CD34- fraction demonstrating that the primary cell population expanded in the MNC fraction are the CD34+ cells. Although the CD34-ve fraction still contained significant numbers of CD34+ cells, the level of expansion was minimal suggesting subsets of CD34+ cells. We hypothesize that selection of the $\mathrm{CB}$ product results in isolation of CD34+ cells expressing higher levels of CD34 and these cells have the highest proliferative potential. 
Table 3 Comparison of Expansion from CB MNC, CD34+ and CD34- Cells. The results presented are for triplicate cultures for each culture condition and are from a single representative experiment of 3 separate experiments

\begin{tabular}{|c|c|c|c|c|}
\hline & & Day 0 & Day 14 & $\begin{array}{l}\text { Fold } \\
\text { expansion }\end{array}$ \\
\hline \multirow{7}{*}{ MNC } & TNC & $2.2 \times 10^{7}$ & $3.4 \times 10^{8}$ & 15 \\
\hline & & $2.2 \times 10^{7}$ & $3.2 \times 10^{8}$ & 15 \\
\hline & & $2.2 \times 10^{7}$ & $4.0 \times 10^{8}$ & 18 \\
\hline & Median & $2.2 \times 10^{7}$ & $3.4 \times 10^{8}$ & 15 \\
\hline & CD34+ cells & $4.5 \times 10^{5}$ & $1.1 \times 10^{7}$ & 25 \\
\hline & & $4.5 \times 10^{5}$ & $1.3 \times 10^{7}$ & 29 \\
\hline & & $4.5 \times 10^{5}$ & $2.0 \times 10^{7}$ & 46 \\
\hline \multirow{9}{*}{ CD34+fx } & Median & $4.5 \times 10^{5}$ & $1.3 \times 10^{7}$ & 29 \\
\hline & TNC & $1.7 \times 10^{5}$ & 2. $1 \times 10^{8}$ & 1,235 \\
\hline & & $1.7 \times 10^{5}$ & $2.9 \times 10^{8}$ & $\mathrm{I}, 705$ \\
\hline & & $1.7 \times 10^{5}$ & $2.3 \times 10^{8}$ & I,352 \\
\hline & Median & $1.7 \times 10^{5}$ & $2.3 \times 10^{8}$ & 1,352 \\
\hline & CD34+ cells & $8.3 \times 10^{4}$ & $4.3 \times 10^{6}$ & 52 \\
\hline & & $8.3 \times 10^{4}$ & $6.2 \times 10^{6}$ & 74 \\
\hline & & $8.3 \times 10^{4}$ & $4.5 \times 10^{6}$ & 54 \\
\hline & Median & $8.3 \times 10^{4}$ & $4.5 \times 10^{6}$ & 54 \\
\hline \multirow{8}{*}{ CD34-fx } & TNC & $2.2 \times 10^{7}$ & $7.1 \times 10^{7}$ & 3 \\
\hline & & $2.2 \times 10^{7}$ & $5.9 \times 10^{7}$ & 3 \\
\hline & & $2.2 \times 10^{7}$ & $8.8 \times 10^{7}$ & 4 \\
\hline & Median & $2.2 \times 10^{7}$ & $7.1 \times 10^{7}$ & 3 \\
\hline & CD34+ cells & $4.0 \times 10^{5}$ & $1.6 \times 10^{6}$ & 4 \\
\hline & & $4.0 \times 10^{5}$ & $1.8 \times 10^{6}$ & 4 \\
\hline & & $4.0 \times 10^{5}$ & $1.6 \times 10^{6}$ & 4 \\
\hline & Median & $4.0 \times 10^{5}$ & $1.6 \times 10^{6}$ & 4 \\
\hline
\end{tabular}

\section{Discussion}

We would propose two potential advantages to the use of co culture for expansion based upon the above results. Firstly the possible enhanced engraftment and secondly the ability to use better matched $\mathrm{CB}$ products that may have a low cell dose. Wagner and colleagues have described the use of two $\mathrm{CB}$ products to provide an increased cell dose; however, the majority of patients receive a 2 antigen miss matched $\mathrm{CB}$ unit. ${ }^{13}$ Better matched $\mathrm{CB}$ units are routinely identified but are not suitable due to low cell doses. The expansion of the better matched $\mathrm{CB}$ units could potentially result in decreased toxicities relating to graft versus host disease (GVHD).

The culture conditions used in this study have been used in previous clinical trials of ex vivo expansion of both $\mathrm{CB}^{10}$ and peripheral blood progenitor cell products (PBPC).$^{14}$ In particular the expanded PBPC products resulted in faster neutrophil engraftment which correlated with the total nucleated cells (TNC) infused. ${ }^{14}$ In addition, we have previously reported that $\mathrm{CD} 34$ selection of frozen $\mathrm{CB}$ products results in low purities and low levels of expansion. ${ }^{11}$ When CB MNC were cultured for 14days, little if any expansion resulted, however, co-culture on MSC resulted in significant expansion of TNC and progenitor cells (CD34+ cells and CFU).$^{11}$ The expanded CB products contain high numbers of neutrophils and promyelocytes and less than $1 \% \mathrm{~T}$ and $\mathrm{B}$ lymphocytes. ${ }^{10,11}$ Although the focus of this study was expansion of TNC, our previous studies have demonstrated that long term repopulating stem cells are decreased following expansion. When ex vivo expanded CB cells were injected into fetal sheep the levels of human engraftment were decreased compared to sheep transplanted with non expanded CB cells. ${ }^{15}$ Based upon these data, the clinical trial design currently being undertaken with co-culture of $\mathrm{CB}$ MNC on MSC, ${ }^{12}$ utilizes two CB products with the expanded $\mathrm{CB}$ product providing enhanced short term engraftment while the un expanded $\mathrm{CB}$ product provides the long term engraftment. This study reported the median time to neutrophil and platelet engraftment of 15 days and 42 days respectively. ${ }^{12}$ The median fold expansion of TNC was 12 fold. This study has led to a phase III trial for evaluation of $\mathrm{CB}$ expansion on MSCs.

A second clinical trial of expanded CB cells is being conducted at the Fred Hutchinson Cancer Center by Dr. Bernstein and colleagues. ${ }^{16}$ This study targets expansion of CD34+ cells using an engineered form of the Notch ligand, Delta 1. Preliminary data was reported at meeting of the American Society of Hematology on the first 6 patients enrolled in this study and reported rapid engraftment with a median time to neutrophil recovery of 14days (range 7-34days).

Taken together these studies demonstrate the potential of expanded $\mathrm{CB}$ cells to provide more rapid engraftment. The data we have presented in this reports expands the range of $\mathrm{CB}$ products that may be used for therapy. The clinical trials described above utilize $\mathrm{CB}$ products with higher cell numbers and typically $\mathrm{CB}$ products with smaller cell doses would not meet minimum cell dose requirements. Testing of this approach will require a clinical trial to evaluate the engraftment potential of these expanded products.

\section{Acknowledgements}

None.

\section{Conflict of interest}

The author declares no conflict of interest.

\section{References}

1. Gluckman E, Devergie A, Bourdeau-Esperou H, et al. Transplantation of umbilical cord blood in Fanconi's anemia. Nouv Rev Fr Hematol. 1990;32(6):423-425.

2. Wagner J. Umbilical cord blood stem cell transplantation. Am J Pediatr Hematol Oncol. 1993;15(2):169-174.

3. Wagner JE, Kernan NA, Steinbuch M, et al. Allogeneic sibling umbilical-cord-blood transplantation inchildren with malignant and non-malignant disease. Lancet. 1995;346(8969):214-219.

4. Wagner JE, Rosenthal J, Sweetman R, et al. Successful transplantation of HLA-matched and HLA-mismatched umbilical cord blood from unrelated donors: analysis of engraftment and acute graft-versus-host disease. Blood. 1996;88(3):795-802.

5. Kurtzberg J, Laughlin M, Graham ML, et al. Placental blood as a source of hematopoietic stem cells for transplantation into unrelated recipients. N Engl J Med. 1996;335(3):157-166.

6. Gluckman E, Rocha V. Donor selection for unrelated cord blood transplants. Curr Opin Immunol. 2006;18(5):565-570. 
7. Gluckman E, Rocha V, Boyer-Chammard A, et al. Outcome of cordblood transplantation from related and unrelated donors. Eurocord Transplant Group and the European Blood and Marrow Transplantation Group. N Engl J Med. 1997;337(6):373-381.

8. Jaroscak J, Martin PL, Waters-Pick B, et al. A phase I trial of augmentation of unrelated umbilical cord blood transplantation with ex vivo expanded cells. Blood. 1998;92(Suppl 1):646a.

9. Stiff P, Pecora A, Parthasarathy M, et al. Umbilical cord blood transplants in adults using a combination of unexpanded and ex vivo expanded cells: preliminary clinical observations. Blood. 1998;92(Suppl 1):646a.

10. Shpall EJ, Quinones R, Giller R, et al. Transplantation of ex vivo expanded cord blood. Biol Blood and Marrow Transplant. 2002;8(7):368376.

11. McNiece I, Harrington J, Turney J, et al. Ex vivo expansion of cord blood mononuclear cells on mesenchymal stem cells (MSC). Cytotherapy. $2004 ; 6(4): 311-317$.
12. de Lima M, McNiece I, Robinson S, et al. Cord-blood engraftment with ex vivo mesenchymal-cell coculture. $N$ Engl J Med. 2012;367(24):23052315 .

13. Barker JN, Weisdorf DJ, DeFor TE, et al. Transplantation of 2 partially HLA-matched umbilical cord blood units to enhance engraftment in adults with hematologic malignancy. Blood. 2005;105(3):1343-1347.

14. McNiece I, Jones R, Bearman SI, et al. Ex vivo expanded peripheral blood progenitor cells provide rapid neutrophil recovery in breast cancer patients following high dose chemotherapy. Blood. 2000;96(9):30013007.

15. McNiece IK, Almeida-Porada G, Shpall EJ, et al. Ex vivo expanded cord blood cells provide rapid engraftment in fetal sheep but lack longterm engrafting potential. Exp Hematol. 2002;30(6):612-616.

16. Delaney C, Brashem-Stein C, Voorhies H, et al. Notch-Mediated Expansion of Human Cord Blood Progenitor Cells Results in Rapid Myeloid Reconstitution in Vivo Following Myeloablative Cord Blood Transplantation. Blood. 2008;112:212. 